\title{
LES DÉFIS MONDIAUX A VENIR EN 2020 ET 2021, 2022
}

\author{
Author / Auteur : \\ Marc LUYCKX \\ Doctor in Theology (Greek \& Russian) \\ marcluyckxghisi66@gmail.com
}

\begin{abstract}
Résumé :
We are living, according to Naomi Klein, a very effective Worldwide Shock Strategy. Indeed, after the covid19 crisis, we could be confronted with an important economic collapse of Banks, moneys, and stock markets. Governments will probably be obliged to install a "Basic Income" for the poorest citizens. To create new money systems for companies and for citizens. And to promote culture in a new and very powerful way. We will also discover after this collapse, that the new circular economy is already functioning very well.

Two scenarios are possible. 1. An extreme but soft political control of the citizens, "Chinese way". And the scenario 2 will be a very important paradigm shift, towards a higher level of consciousness, with a new spiritual dimension. This second scenario becomes more and more possible.
\end{abstract}

Keywords / Mots-clés :

shock strategy, economic collapse, basic income, culture, 2 scenarios

\section{PARTIE 1 : UNE STRATEGIE DE CHOC BIEN REUSSIE : LA CRISE SERA AUSSI ECONOMIQUE}

Noami Klein a écrit en 2008, La stratégie du choc. Elle explique que ce choc politique et économique organisé vise d'abord à sidérer la population. Et il permet ensuite aux politiques de faire passer des lois très autoritaires, sous le prétexte de protéger la population apeurée.

Notre hypothèse est que nous sommes en train de vivre une "stratégie du choc" admirablement bien organisée. Elle est en effet parvenue à arrêter l'économie de 4 milliards de citoyens sur la terre. Remarquable réussite.

Et donc nous ne sommes probablement en train de vivre que la première phase présentée comme une "Pandémie". La phase qui vient ensuite sera une crise économique de très grande ampleur.

\section{Crise économique majeure possible en 2020 (ou 2021)}

Il y a en effet $40 \%$ de possibilités qu'une crise économique majeure touche les États-Unis et l'Europe, mais aussi le reste du monde selon des modalités différents. Et cela dans les mois ou les années à venir. Le FMI nous en a avertis depuis 2018.

1. Crash monétaire: (dévaluation forte mais silencieuse du $\$$ et de l'€. C'est-à-dire perte de $25 \%$ ou plus, du pouvoir d'achat des citoyens en quelques jours).

2. Crash boursier: Des acteurs importants vont être ruinés. Et aussi crise majeure de la valeur en Bourse des entreprises. Comment la mesurer, si la Bourse s'écroule?

3. Et crash bancaire : $30 \%$ des banques EU et USA sont en danger.

C'est donc l'ensemble du système économique qui pourrait s'écrouler.

\section{Faillite possible de $30 \%$ des Banques Européennes et US et "BAIL-IN" (vol des citoyens)}

Il semble que $30 \%$ des banques européennes pourraient faire faillite s'il y a trop de remous financiers. La banque qui semblerait la plus en danger en Europe serait la "Deutsche Bank" qui est alourdie de 
nombreux crédits frauduleux, dont elle ne parvient pas à se débarrasser. Et un effet domino pourrait faire crouler de nombreuses Banques européennes.

Or dans nos pays et au niveau européen, la loi autorise les banques à appliquer le "Bail-in". Le Bail-in a déjà été pratiqué à Chypre. Les rideaux des banques se sont fermés un jour. Et le lendemain les banques ont rouvert, et les citoyens qui avaient $500.000 €$ ou plus sur leur compte se sont rendu compte que la Banque avait pompé $400.000 €$, puisque la loi européenne et nationale ne garantit que $100.000 €$ par compte en cas de crise bancaire.

Pour les PME qui ont leurs réserves et leurs actifs en banque, cela signifierait la faillite, et le chômage massif.

\section{Faillites de nombreuses entreprises surtout PME.}

Il est probable que de nombreuses entreprises vont disparaître après le confinement. Surtout les PME qui constituent le tissu économique principal de nos économies européennes, et le plus grand pourvoyeur d'emplois.

\section{Possibilité de Chômage pour $40 \%$ des citoyens}

On pourrait donc avoir un niveau de chômage stable et exceptionnellement haut de 30 à $40 \%$, en Europe et aux USA.

Il faut ajouter aussi que le monde politique n'ose pas dire la vérité au sujet de l'emploi post-industriel. Il n'y aura pas d'emploi pour tout le monde dans l'économie immatérielle. Cette vérité change radicalement toute la politique des "Pôles emplois" qui sont basés sur l'hypothèse (industrielle) qu'il est possible à tout le monde de trouver un emploi. Ce monde-là est en train de disparaître. D'où l'importance cruciale du débat sur le Revenu Universel et social (cf. $2^{\mathrm{e}}$ partie de cet article).

\section{Mort de nombreuses structures pyramidales}

Ces moments de crise vont ressembler plus à une mutation mondiale à partir de l'Occident. Et on risque de voir s'effondrer bon nombre de structures pyramidales qui ne fonctionnent plus correctement dans le monde actuel. Entreprises, Syndicats, églises, Ordres des médecins, des Avocats, Conseils Scientifiques, etc. Elles ne parviennent pas à répondre aux exigences de transparence, de soutenabilité, d'efficacité en intelligence collective, et donc de sens. Elles meurent parce qu'elles ne font plus sens.

\section{Situation catastrophique dans le Tiers Monde}

Inutile de dire que ce choc économique et financier aurait un impact très négatif sur les populations pauvres du Tiers monde. Or cette population même si elle a diminué est encore de plusieurs milliards de citoyens.

\section{CONCLUSION : LES COLLAPSOLOGUES ONT RAISON... EN PARTIE}

Oui, les collapsologues (Servigne \& Stevens, 2015) ont raison, du moins en partie. Un effondrement important de l'économie et de la civilisation Occidentale est un des scénarios possibles. Mais en même temps, il faut bien comprendre que c'est au moment où la plaque industrielle va s'effondrer qu'on va découvrir tout à coup la nouvelle plaque tectonique post-industrielle. Une nouvelle logique économique et sociale mondiale qui existe et fonctionne déjà, même si elle n'est pas mise en valeur dans les médias officiels. 


\section{$2^{\circ}$ PARTIE LA NOUVELLE PLAQUE TECTONIQUE : LA CIVILISATION SOUTENABLE ET JUSTE}

\section{Revenu Universel Social}

Une guerre civile de Gilets Jaunes devant les supermarchés, pour la nourriture, est un scénario possible. La seule solution pour les Gouvernements Occidentaux sera peut-être d'établir rapidement une modalité de revenu universel social, qui serait modulé selon les revenus. Si vous gagnez plus de $3000 €$ par mois vous n'en avez pas besoin. On peut en effet s'arranger aussi pour que le revenu social des riches soit récupéré par la fiscalité. Les modalités seront à perfectionner progressivement.

Mais ce Revenu Universel suppose un système social et une citoyenneté complètement nouveaux.

Il est important de noter que le concept même de Revenu universel implique une redéfinition importante de la citoyenneté, qui n'est plus définie par l'emploi, comme dans l'ère industrielle, mais par la dignité intrinsèque de tout citoyen. C'est un nouveau paradigme sociétal qui devra se mettre en place. Et cela va prendre du temps.

\section{Innovations monétaires pour les entreprises et pour les citoyens}

\section{Un WIR européen}

En Suisse en 1928, il y a cent ans, les grosses entreprises Winterthur (assurances) Migros (alimentation) et autres décidèrent de créer une monnaie entre entreprises qu'elles appelèrent le "WIR ${ }^{1 "}$. Cette monnaie leur a permis de passer à travers la crise financière de 1929, sans trop de dégâts. Il est donc important que soit créée rapidement une monnaie complémentaire européenne au service des entreprises.

\section{Monnaies citoyennes}

Mais il faudra aussi probablement créer des systèmes monétaires citoyens ou de monnaies complémentaires. Ces systèmes seront aussi très importants comme liant social. Les villes en transition comme Totnes l'ont compris (Hopkins, 2010). Et il existe des centaines de monnaies de ce type en UE et USA. Dans ce domaine monétaire, on trouvera une importante source d'information dans le Rapport du Club de Rome au Parlement Européen en 2012 écrit par Bernard Lietaer (2012).

\section{La Découverte que l'économie circulaire fonctionne déjà... depuis longtemps}

Le centre de ce nouveau narratif est que :

- Les valeurs dominantes ne sont plus liées aux axiomes de l' "Homo economicus industrialis". Cet homo economicus est défini en effet, comme égoïste. Il ne se préoccupe ni du social ni de l'environnement, ni du futur. Car la main invisible du marché, transformera la somme de ces égoïsmes en un marché équilibré.

- Les nouvelles valeurs dominantes : le nouvel "Homo economicus immaterialis" peut aussi se comporter de manière solidaire et il respecte et régénère l'environnement. Il promeut des valeurs plus sages et adultes (A. Piet). Il crée le nouveau narratif soutenable. Et ces axiomes nouveaux s'installent au cœur de la nouvelle économie, qui fonctionne déjà.

- La Nouvelle création de valeur économique. La nouvelle création de valeur consiste à appliquer de la connaissance à de la connaissance pour créer de la connaissance ${ }^{2}$ (exemples : Facebook, AirB\&B, Uber, Amazon, etc.). Ceci signifie que l'outil de travail n'est plus l'usine, mais l'humain qui crée de la nouvelle connaissance avec son intelligence, son intuition et son âme. C'est une nouvelle définition de l'outil de travail. Le management devient donc humaniste par obligation, même si de nombreuses entreprises essaient de tricher.

\footnotetext{
1 "WIR" signifie "Nous" en allemand. Le Centre de WIR se trouve à Zurich.

${ }^{2}$ Cette définition a été expliquée et inventée par Peter Drucker en 1993 : L'après capitalisme : la métamorphose de cette fin de siècle" Dunod 1993.
} 
Les acquis principaux deviennent majoritairement immatériels. Donc c'est l'ensemble des instruments de mesure qui doivent être totalement repensés (à partir des "actifs immatériels").

- Nouveau concept de technologie : Comme disait Steve JOBS le fondateur d'Apple, la technologie est là pour être "Human Friendly and beatiful". Le contraire de la logique industrielle où c'est la machine qui domine l'humain. C'est pourquoi la "Singularity University" créée par R. Kurzweil (1999 ; 2005) à Palo Alto (Californie) est déjà obsolète, car elle est restée coincée dans le paradigme industriel matérialiste.

\section{Le meilleur Rapport d'un Gouvernement (FR).}

Au plan européen, je ne connais qu'un seul rapport gouvernemental qui décrit le changement de paradigme économique et la nouvelle logique économique circulaire et immatérielle. C'est le rapport commandé par Ségolène Royal, alors Ministre de l'environnement, et écrit par Corinne Lepage, en 2015. Voici une partie du résumé du rapport donné à la p.16 de mon édition :

"... La nouvelle économie repose sur un changement complet de paradigme, dans lequel le numérique rend le marché plus productif, plus compétitif, davantage basé sur des valeurs d'échange et de partage, de réappropriation de l'environnement et de l'économie locale"

"... Le capital social est aussi vital que le capital financier, l'accès est aussi important que la propriété, la durabilité remplace le consumérisme, la coopération est aussi cruciale que la concurrence, et la valeur partageable sur les communaux collaboratifs, vient de plus en plus souvent compléter la valeur d'échange sur le marché capitaliste" (Rifkin).

"Nous avons tout pour réussir. Notre pays peut faire état de centaines de réussites à son actif, menées par des citoyens, des entreprises (surtout PME) et des territoires qui ont parfaitement analysé le Nouveau Monde et la manière de s'y insérer..."

Nous pouvons faire beaucoup mieux, c'est-à-dire changer d'échelle et faire de la transition vers cette nouvelle économie un projet de société à part entière, capable de réunir, bien au-delà des divisions partisanes, et dans le sillage de tous les pionniers, de très nombreux acteurs qui ne demandent qu'à le soutenir."

Ce rapport exceptionnel analyse aussi les freins institutionnels qui empêchent cette nouvelle économie de se développer au-delà du niveau régional, qui fonctionne déjà très bien dans certaines régions, par exemple en Aquitaine.

\section{Un livre qui ose repenser les axiomes de base de l'économie mondiale : Aurélie PIET.}

Je ne connais pas beaucoup de livres qui osent repenser les axiomes de base de l'économie mondiale. Une jeune économiste bordelaise, Aurélie Piet (2019) a brillamment réussi ce défi. Et elle décrit les nombreux économistes, y compris certains prix Nobel qui font ce travail de repensée en profondeur. Mais c'est Mme Piet qui a la vision la plus globale et la plus crédible, car elle lie intelligemment la repensée et la recréation de l'économie à celle de notre civilisation post-industrielle et transmoderne.

Elle trace un itinéraire de transformation positive de l'économie mondiale, même si on nous abreuve de scénarios négatifs dans les médias.

\section{Les nouvelles entreprises libérées}

Alors que de nombreuses entreprises vont disparaître dans les années à venir, d'autres se créent ou se transforment en profondeur. Avec la société industrielle disparaît aussi la vision de l'entreprise comme machine, comme mécanique, dans laquelle l'humain n'est qu'un rouage ${ }^{3}$.

Et on "découvre" tout à coup que l'entreprise est composée d'humains (Laloux, 2015) que l'on peut inviter à créer en "intelligence collective" (Heinrich, 2019). On change le "narratif" de l'entreprise. Et le nouveau narratif est basé sur les nouvelles valeurs dominantes (du nouveau monde/paradigme)

- La soutenabilité et la restauration de l'environnement

- La solidarité sociale au plan local, national, européen et mondial.

\footnotetext{
${ }^{3}$ Cette vision a été magnifiquement illustrée par Charlie Chaplin se représentant dans une énorme roue dentée.
} 
- La participation active et créatrice des citoyens en intelligence collective.

- Le profit est une conséquence importante qui révèle que tout est $\mathrm{OK}$.

\section{Le choc comme opportunité exceptionnelle de changement positif}

Nous avons parlé de la réussite exceptionnelle de la stratégie du choc actuelle. Mais ce choc sur notre monde Occidental et mondial, est aussi une opportunité exceptionnelle de changement en profondeur. Peut-être en effet, que ce n'est qu'à des moments pareils que les structures des entreprises et de la politique peuvent être modifiées en profondeur et très positivement. C'est ce que suggère Naomi Klein, et nous partageons cette analyse.

\section{La Nouvelle mission des entreprises}

Ceci signifie aussi que les entreprises ont une nouvelle mission. Elles sont potentiellement des acteurs importants dans la construction de cette nouvelle économie du Nouveau Monde. C'est ce qui explique le succès d'initiatives de formation comme "Ticket For Change" à Paris.

Les entreprises peuvent être un élément important dans l'évolution positive et constructive de l'humanité au $21^{\circ}$ siècle.

\section{Le Rôle central de la culture comme nourriture indispensable de la créativité humaine dans la nouvelle économie immatérielle.}

Nos gouvernements hésitent à investir massivement pour "sauver" le monde culturel, parce qu'ils sont malheureusement encore scotchés à la vielle vision industrielle, matérialiste, et mécaniciste, dans laquelle la culture est la cerise sur le gâteau...si tout va bien.

Mais dans la société immatérielle où la créativité humaine est l'outil de travail principal, la culture devient absolument indispensable car elle alimente la créativité humaine. Richard Florida (2002) a déjà expliqué il y a 20 ans, combien la culture a profondément changé de rôle dans la nouvelle économie de la créativité humaine.

Il sera donc crucial pour les gouvernements éclairés de mettre une priorité absolue sur le financement et l'autonomisation de toute l'activité culturelle. C'est plus important que de "sauver" des compagnies aériennes moribondes.

\section{CONCLUSION : LA NOUVELLE CIVILISATION : DEUX SCENARIOS}

Je crois que les circonstances exceptionnelles que nous vivons nous approchent de deux scénarios possibles.

Le premier scénario serait une civilisation du contrôle de plus en plus total mais soft, des citoyens du monde. Et ce scénario a été très bien annoncé par Huxley [2002] et Orwell [1990]. Et en plus "La stratégie du choc" annoncée par Naomi Klein, semble avoir été appliquée avec grand succès.

Vers où irions-nous dans ce premier scénario ? Et ici le modèle contemporain le plus exemplatif est le modèle chinois actuel, qui contrôle chaque citoyen électroniquement (contrôle facial) en lui donnant une approbation rouge jaune ou verte sur son portable. Seul le signal vert permet de voyager et de faire des achats des emprunts et des investissements. L'émission récente (fin avril 2020) sur ARTE l'a bien décrit ${ }^{4}$.

Et ce régime de contrôle n'hésite pas à organiser l'emprisonnement de millions de citoyens Ouïgours, uniquement parce qu'ils sont musulmans. Et ils sont enfermés dans des usines-prisons où ils travaillent en travaux forcés pour nos multinationales occidentales.

Le second scénario s'enracine dans la découverte progressive par les citoyens du monde que ce monde du contrôle Orwellien n'a, en fait, aucune vision d'avenir soutenable (No future), sauf peut-être de faire disparaitre quelques milliards d'humains "en trop" (Bill Gates). Or tout projet politique sans vision d'avenir est condamné à mourir, comme ce fut le cas pour le puissant empire romain.

${ }^{4}$ ARTE 22 avril 2020 "Tous surveillés : 7 milliards de suspects". https://www.youtube.com/watch?v=8wN3emyA-ew 
Et c'est donc en se mettant debout ensemble, et en promouvant et en mettant en oeuvre une nouvelle vision d'avenir symbolisée par des nouvelles valeurs de vie, de soutenabilité, de solidarité, de justice et de liberté, que l'Humanité sera à même de créer une nouvelle civilisation plus adulte, plus soutenable plus éthique et plus spirituelle. Et certains sociologues comme Paul H. Ray (\& Anderson, 2001) avancent l'hypothèse que les "créateurs culturels" annoncent déjà les valeurs de la civilisation de demain. Ils pourraient être entre un et deux milliards sur notre planète. C'est important.

Et l'économie circulaire avec le changement de vision qu'elle promeut, est un des facteurs qui favorise l'évolution positive, et l'élévation de notre niveau de conscience.

Cette nouvelle civilisation d'un niveau plus élevé a été annoncée par Jésus (le Royaume des Cieux), par Teilhard de Chardin (La Noosphère), et par Aurobindo et la Mère, en Inde en 1950.

C'est le second scénario qui est en train de s'établir irrésistiblement et en douceur, aidé peut-être par des forces spirituelles puissantes.

Il est permis d'espérer.

\section{BIBLIOGRAPHIE}

DRUCKER, Peter (1993). L'après capitalisme: la métamorphose de cette fin de siècle". Paris: Dunod.

FLORIDA, Richard (2002). The rise of the creative class and how it is transforming work, leisure, community and everyday life.

HEINRICH, Joseph. (2019). L'Intelligence Collective.

HOPKINS, Rob (2010). Manuel de Transition.

HUXLEY, Aldous (2002). Le meilleur des Mondes. Paris : Poche.

KLEIN, Naomi (2008). La stratégie du choc : La montée d'un capitalisme du désastre. Arles : Actes Sud.

KURZWEIL, Ray (1999). Serons nous tous immortels? [The age of spiritual Machines]. éd.2006.

KURZWEIL, Ray (2005). The singlularity is near. When Humans transcend biology.

LALOUX, Frédéric (2015). Reinventing Organizations. Vers des communautés de Travail Inspirées.

LIETAER, Bernard (2012). Halte à la Toute-Puissance des Banques: Rapport du Club de Rome au Parlement Européen. Paris : Odile Jacobs.

ORWELL, Georges (1990). 1984. Paris : Poche.

PIET, Aurélie (2019). Quand l'Homo economicus saute là l'élastique sans élastique. Paris : Plon.

RAY, Paul H. \& ANDERSON, Sherry (2001). L'émergence des créatifs culturels : Enquête sur les acteurs d'un changement de société. Paris : Yves Michel.

SERVIGNE, Pablo \& STEVENS, Raphaël (2015). Comment tout peut s'effondrer. 